[0212-7199 (2007) 24: 8; pp 375-378] ANALES DE MEDICINA INTERNA Copyright (C) 2007 ARAN EDICIONES, S.L.

AN. MED INTERNA (Madrid) Vol. 24, N. ${ }^{\circ} 8$, pp. 375-378, 2007

\title{
Optimización del tratamiento antibiótico mediante la participación de expertos en antibióticos
}

\author{
A. RAMOS MARTÍNEZ, E. MUÑEZ RUBIO, A. SANTIAGO PÉREZ ${ }^{1}$,E. GARCÍA \\ SANZ, M. MANSO MANRIQUE ${ }^{1}$, A. TORRALBA ARRANZ ${ }^{1}$, A. ASENSIO VEGAS ${ }^{2}$ \\ Unidad de Enfermedades Infecciosas. Servicio de Medicina Interna. ${ }^{I}$ Servicio de Farmacia. \\ ${ }^{2}$ Servicio de Medicina Preventiva. Hospital Universitario Puerta de Hierro. Universidad \\ Autónoma. Madrid
}

\begin{abstract}
ANTIBIOTIC TREATMENT OPTIMIZATION BY MEANS OF ANTIBIOTIC TREATMENT EXPERTS PARTICIPATION
\end{abstract}

\section{RESUMEN}

Antecedentes: Los antibióticos representan un porcentaje elevado del gasto de farmacia de un hospital. Una elevada proporción de las prescripciones se consideran inapropiadas. La ayuda prestada por infectólogos con una formación extensa en el manejo de los antibióticos podría mejorar la prescripción de este tipo de medicamentos.

Métodos: Revisión diaria de las prescripciones antibióticas realizadas en cuatro salas de hospitalización de varias especialidades quirúrgicas. Examen diario de los casos que cumplían algunos de los signos de sospecha de prescripción inadecuada. Si existían casos sugestivos de este tipo de prescripción se efectuaba una recomendación a los médicos prescriptores. La intervención se realizó durante 4 meses. Se compararon los resultados con un periodo similar del año anterior.

Resultados: Se revisaron 562 tratamientos en 393 pacientes. Se realizaron 524 recomendaciones (el 90\% fueron aceptadas). Se logró una reducción importante de prescripciones consideradas inadecuadas. Se consiguió una disminución del gasto en antibióticos de $29.363 €(7.240$ $€ /$ mes), lo que supone un ahorro de 2,35€/cama de hospitalización/día. No hubo diferencias estadísticamente significativas ni en la en mortalidad ni en el aislamiento de bacterias nosocomiales resistentes

Conclusiones: Empleando un programa de asesoramiento sobre el tratamiento antibiótico se consiguió una mejor utilización y una disminución del gasto en antibióticos, sin variaciones en la mortalidad. El programa fue muy bien aceptado por los médicos de los servicios implicados.

PALABRAS CLAVE: Antibióticos. Programa de control. Gasto económico. Infección nosocomial.

\section{ABSTRACT}

Background: Antibiotics account for a high percentage of hospital pharmacy expenses. An elevated proportion of the prescriptions are considered inappropriate. Infectious diseases specialist could help other physicians in improving antibiotics prescriptions

Methods: Treatments in all patients hospitalized in four surgical wards were checked daily and recommendations were made in cases with signs of inadequate antibiotics prescriptions. The program was carried out during for 4 months and the results were compared with a similar period of the previous year.

Results: 562 treatments of 393 patients were reviewed. Five hundred twenty four recommendations were made $190 \%$ of them were accepted). There was a significant reduction in the number of inadequate prescriptions. Antibiotic expenditure decreased by 29,262 euros $(7,240$ euros/month), implying a reduction of 2.35 euros/hospitalizationbed/day. There were no statistically significant differences in the amount of hospital resistant bacteria nor in mortality between the two periods.

Conclusions: Following application of a non-compulsory control program, antibiotic prescription improved and expenditure decreased, with no change in mortality. Acceptation of the program by the physicians of the departments implicated was favourable.

KEY WORDS: Antibiotics. Control Program. Hospital Costs. Nosocomial infection.

Ramos Martínez A, Muñez Rubio E, Santiago Pérez A, García Sanz E, Manso Manrique M, Torralba Arranz A, Asensio Vegas A. Optimización del tratamiento antibiótico mediante la participación de expertos en antibióticos. An Med Interna (Madrid) 2007; 24: $375-378$.

\section{INTRODUCCIÓN}

El gasto farmacéutico hospitalario de antimicrobianos supera el coste de cualquier otro grupo terapéutico (1). La deficiente calidad de la prescripción antibiótica e incluso la inexistencia de una indicación clara para su administración constituyen problemas relevantes en nuestras instituciones sanitarias (2-4). El empleo inadecuado de antibióticos pro- mueve la selección de cepas de bacterias resistentes y merma los recursos económicos de nuestro sistema sanitario $(3,5)$. En los últimos años se han efectuados diversas experiencias cuyo objetivo ha sido mejorar la utilización de los antibióticos en los hospitales $(2,3,6)$. Las iniciativas basadas en la colaboración entre servicios clínicos y en la asunción compartida de la responsabilidad de cada caso puede incrementar sus posibilidades de éxito $(2,3)$. 
En los últimos meses se ha llevado a cabo una experiencia en un hospital de Madrid cuyo objetivo era conocer si la colaboración estrecha entre infectólogos y otros especialistas podría mejorar significativamente la calidad de la prescripción antibiótica.

\section{MATERIAL Y MÉTODO}

El Hospital Universitario Puerta de Hierro es un hospital terciario de Madrid que cuenta con 500 camas. Fueron seleccionadas las salas de hospitalización de 4 servicios quirúrgicos que consultan a la Unidad de Infecciosas para manejar los casos complejos de infecciones. Eran los servicios de Cirugía General (46 camas), Cirugía Ortopédica y Traumatología (32 camas), Neurocirugía (16 camas) y Cirugía Cardiovascular (10 camas).

Para llevar a cabo el trabajo dos médicos de la Unidad de Enfermedades Infecciosas dedicaron 3 horas al día a su desarrollo. Los servicios seleccionados aceptaron participar en el estudio señalándose específicamente una responsabilidad compartida en los casos atendidos.

El Servicio de Farmacia informaba todos los días laborables de aquellos pacientes que presentaba alguna circunstancia que sugería la existencia de una prescripción antibiótica que debería ser revisada por presentar algún signo de posible prescripción incorrecta o al menos mejorable en cuanto a su calidad. Los pacientes cuyo tratamiento debía ser revisado debían cumplir una o más de las siguientes características.

-Empleo de antibiótico por vía intravenosa durante más de 5 días.

-Empleo de antibiótico por vía oral durante más de 10 días.

- Administración de más de dos antibióticos simultáneamente.

-Empleo de aminoglucósidos en una pauta diferente de la monodosis diaria.

- Empleo de quinolonas por vía intravenosa.

-Empleo de glicopéptidos, linezolid, carbapenemas y piperacilina/tazobactam

El médico asignado revisaba la hoja de tratamiento, historia clínica y visitaba al paciente antes de hacer una recomendación terapéutica. Se perseguía favorecer el empleo de terapia secuencial (vía intravenosa a vía oral), adecuar el tratamiento antibiótico a los resultados del antibiograma, reducir el empleo de antibióticos antipseudomónicos si no eran necesarios, limitar el empleo de piperazilina-tazobactam, y carbapenémicos para el tratamiento de infecciones nosocomiales, utilización de la vía oral en antibióticos que presenten una adecuada biodisponibilidad y suspensión del tratamiento en casos innecesarios o no justificados. Tras realizar una entrevista con el médico responsable se consignaba en la hoja de recogida de datos si se iban o no a seguir las recomendaciones realizadas.

Los resultados se introdujeron en una base de datos informatizada para su posterior análisis.

La intervención del programa se realizó entre el 15 de marzo y el 15 de julio de 2006. Se estudió la evolución del consumo de los antibióticos que determinaron su revisión y los antibióticos empleados en la sustitución de los mismos. Estos son imipenem, meropenem, piperacilina/tazobactam, aminoglucósidos, teicoplanina, vancomicina, ciprofloxacina, levofloxacina y linezolid. También se comparó el coste eco- nómico del total de antibióticos durante el periodo de estudio con un periodo similar correspondiente al año anterior

El consumo de antibióticos se midió en dosis diarias definidas (DDD) por cada 100 días de estancia hospitalaria, según los criterios definidos por la Organización Mundial de la Salud (OMS) (7). El gasto económico se obtuvo del Servicio de Farmacia. El número de ingresos por servicio en cada periodo fueron facilitados por la unidad de gestión clínica del hospital.

\section{RESULTADOS}

Durante los 4 meses de desarrollo del programa se revisaron 562 tratamientos, pautados a 393 pacientes, que presentaban alguno de los criterios sugestivos de posible tratamiento inadecuado. Se realizaron un total de 269 recomendaciones (en el 47,9\% de los tratamientos revisados) al considerar que el tratamiento actual no era el más adecuado. La distribución de los motivos de las recomendaciones realizadas se recoge en la tabla I. Se aceptaron el $90 \%$ de las recomendaciones realizadas.

\section{TABLA I}

\section{MOTIVO PRINCIPAL DE LAS RECOMENDACIONES} REALIZADAS

\begin{tabular}{lcc}
\hline & N & Porcentaje \\
\hline Prescripción de un antibiótico restringido & 262 & 46 \\
Piperacilina /tazobactam & 81 & 31 \\
Imipenem & 71 & 27 \\
Vancomicina & 71 & 27 \\
Linezolid & 16 & 6 \\
Teicoplanina & 13 & 5 \\
Meropoenem & 10 & 4 \\
Antibiótico por vía endovenosa durante más de 5 días & 103 & 19 \\
Prescripción de 2 antibióticos & 104 & 18 \\
Quinolona por vía endovenosa & 71 & 13 \\
Antibiótico por vía durante más de 10 días & 22 & 4 \\
\hline
\end{tabular}

Se realizaron 362 cambios en las 269 intervenciones (1,34 intervenciones por paciente) En 250 ocasiones se procedió a suspender el antibiótico (69\%), en 60 casos el antibiótico se cambió de vía endovenosa a vía oral (17\%) y en 52 ocasiones (14\%) se procedió a prescribir un nuevo antibiótico

Las DDD para cada antibiótico durante los 2 periodos de tiempo considerados figuran en la tabla II.

Durante el primer periodo hubo 1235 ingresos y 13976 días de estancia hospitalaria y durante el segundo (periodo de intervención) 1.173 ingresos y 13.039 estancias hospitalarias. La diferencia en el coste de los antibióticos empleados durante los periodos analizados en los servicios estudiados figura en la tabla II. Hubo una disminución del gasto de 29.363,9 $€$ (4.914.430 pesetas.) en comparación con el mismo periodo del año anterior. Esta cifra supuso un ahorro de 2,35 €/cama de hospitalización convencional/día, en el global de los servicios implicados en el programa.

Los casos de infección o colonización por Staphylococcus aureus resistente a meticilina, por Pseudomonas spp. productoras de carbapenemasa o enterobacterias resistentes a beta- 


\begin{tabular}{|c|c|c|c|c|}
\hline \multicolumn{5}{|c|}{ TABLA II } \\
\hline \multicolumn{5}{|c|}{$\begin{array}{l}\text { DIFERENCIA ENTRE EL NÚMERO DE DOSIS DIARIAS } \\
\text { DEFINIDAS DE ANTIBIÓTICOS POR } 100 \text { DÍAS DE } \\
\text { HOSPITALIZACIÓN Y COSTE ENTRE AMBOS PERIODOS }\end{array}$} \\
\hline & $\begin{array}{l}\text { Control } \\
15 \text { marzo } 2005 \\
15 \text { julio } 2005\end{array}$ & $\begin{array}{l}\text { Intervención } \\
15 \text { marzo } 2006 \\
15 \text { julio } 2006\end{array}$ & Diferencia & Porcentaje \\
\hline Piper-tazobactam & 2,63 & 2,95 & $+0,32$ & +13 \\
\hline Cloxacilina & 0,34 & 5,07 & $+4,73$ & +1391 \\
\hline Imipenem & 3,98 & 3,86 & $-0,12$ & -3 \\
\hline Meropenem & 1,73 & 0,8 & $-0,93$ & -53 \\
\hline Gentamicina & 1,37 & 2,29 & 0,92 & +67 \\
\hline Teicoplanina & 1,42 & 0,47 & $-0,93$ & -65 \\
\hline Vancomicina & 3,52 & 4,1 & $+0,58$ & +16 \\
\hline Ciprofloxacino VO & 2,56 & 3,14 & $+0,58$ & +23 \\
\hline Ciprofloxacino EV & 2,46 & 3 & $+0,54$ & +22 \\
\hline Levofloxacino VO & 6,65 & 4,38 & $-2,27$ & -34 \\
\hline Levofloxacino EV & 2,44 & 1,59 & $-0,85$ & -34 \\
\hline Linezolid & 0,56 & 0,94 & $+0,38$ & +67 \\
\hline Resto & 46,58 & 47,36 & $+0,78$ & $+1,7$ \\
\hline Total & 76,24 & 79,95 & $+3,71$ & $+4,8$ \\
\hline Coste económico (€) & ) 126370 & 97007 & -29.363 & $-23,2$ \\
\hline
\end{tabular}

VO: administración del antibitiótico por vía oral; EV: administración del antibiótico por vía endovenosa.

lactamasas fueron 13 durante 2005 y 11 durante el 2006. La mortalidad fue del 2,3\% durante primer periodo (año 2005) y del 3,2\% durante el segundo (años 2006; $\mathrm{p}=0,279$ ).

\section{DISCUSIÓN}

La preocupación por los efectos negativos del uso inadecuado de los antibióticos en el ámbito hospitalario ha motivado el desarrollo de programas cuya finalidad es mejorar la calidad de la prescripción $(8,9)$. Una variedad de estos programas consiste en establecer una lista de antibióticos restringidos cuya administración requiere una autorización previa $(3,6,7,10,11)$. Uno de los problemas de este enfoque es que el clínico prescriptor puede contemplarlo como una mera traba burocrática, pudiendo existir, además, el riesgo de atribuir a una determinada restricción antibiótica una potencial mala evolución de un caso particular $(2,12)$. Las actuaciones no impositivas para mejorar el uso antibiótico presentan, sin embargo, un perfil más adecuado para su puesta en práctica $(2,3)$. El acercamiento hacia el médico prescriptor de otro compañero (un infectólogo habitualmente) sugiriendo un cambio en el antibiótico junto a un implicación clínica directa en el caso puede mejorar los resultados (2). El experto en antibióticos permite aportar una mayor sensibilidad y seguridad en la interpretación de los cultivos y resultados del antibiograma, así como una mayor seguridad en el empleo por vía oral de los antibióticos, gracias al mejor conocimiento (13) de aspectos farmacocinéticos y farmacodinámicos de los mismos (14). En nuestra experiencia, al igual que en otros trabajos previos, el acercamiento hacia el médico prescriptor de un modo no impositivo, favoreciendo la responsabilidad compartida sobre la evolución del enfermo, ha tenido una acogida muy favorable $(2,3,13,15)$. También es imprescindible la colaboración de los servicios de farmacia hospitalaria para proporcionar la información sobre a que se sustenta este tipo de estudios $(2,3)$.

Este tipo de actuación es una de las razones de la existencia de las unidades de infectología en los hospitales generales $(12-14,16,17)$. Nuestra intervención no se asoció a ningún dato que haga sospechar peor evolución de los pacientes, ya que ni la mortalidad ni la incidencia de de infecciones por bacterias resistentes durante el periodo de estudio aumentaron significativamente.

Como se puede observar en la tabla II, se produjo una reducción en el empleo de carbapenémicos, especialmente de meropenem durante el segundo periodo. Esta reducción se logró principalmente a expensas de la limitación en el empleo de estos antibióticos para infecciones adquiridas en la comunidad. También es destacable la reducción de teicoplanina respecto a vancomicina justificable por la elección más frecuente de este segundo antibiótico por su menor coste y la posibilidad de medir sus niveles con carácter rutinario (18). Hay que reconocer, sin embargo que en el caso de linezolid se ha objetivado un incremento en su empleo relacionados con casos de alergia a glicopéptidos o dificultad en la obtención de una vía venosa, que podrían tener un componente coyuntural. El incremento en la utilización de cloxacilina estuvo originado en la sustitución de un glicopéptido en casos de infección por cocos grampositivos sensibles a betalactámicos. Este incremento ofrece una idea de los beneficios potenciales de este tipo de iniciativas $(2,3)$.

El desplazamiento de prescripciones parenterales a formas orales permite conseguir un importante ahorro sin que se tenga que producir una disminución semejante en las DDD (2). Utilizar la vía oral desde el principio es posible con algunos fármacos con excelente biobisponibilidad como clindamicina, metronidazol o fluoroquinolonas $(2,14,19,20)$.

Extendiendo los datos obtenidos en el trabajo al resto de 1 hospital, se estima un ahorro potencial de $440.445 €$ al año (equivalente a 73 millones y medio de pesetas) en relación con la prescripción antibiótica sin asesoramiento. A este ahorro que habría que restar el coste del personal encargado de llevar a cabo el programa.

Así, desde un punto de vista exclusivamente económico, nuestra experiencia se muestra como una intervención muy eficiente. Con este tipo de iniciativas, no obstante, también se pueden conseguir una serie de ahorros económicos indirectos, más difíciles de determinar como podrían ser una disminución de días de hospitalización al realizar terapia secuencial que se puede completar de manera ambulatoria, una disminución de infecciones de catéteres intravenosos al disminuir los días de implantación de los mismos, disminución de episodios de diarrea por Clostridium difficile y de sobreinfecciones fúngicas entre otros.

Entre las limitaciones del estudio destaca que no se trata de un ensayo prospectivo de comparación de dos cohortes (expuesta y no expuesta a la intervención), sino que se comparó el efecto de la intervención con los parámetros del año previo a su realización. De modo que la reducción encontrada en el gasto, en el consumo de antibióticos y en la incidencia de infecciones nosocomiales pudiera deberse a factores ajenos a los de la propia intervención. También conviene destacar que la efectividad de este tipo de medidas tiende a disminuir cuando se suspende la intervención (3)

En conclusión, nuestro estudio muestra que en un entorno hospitalario un programa de asesoramiento sobre el uso de 
antibióticos puede ser muy bien aceptado por los profesionales sanitarios y permite obtener una reducción significativa en el gasto de antibióticos y una mejor utilización de los mismos. Sería recomendable una mayor implantación en España, en unidades de hospitalización convencional, de estos programas de control de tratamiento antimicrobiano.

\section{Bibliografía}

1. John JF, Fishman NO. Programmatic role of the infectious diseases physician in controlling antimicrobial costs in the hospital. Clin Infect Dis 1997; 24: 471-85.

2. Cobo Reinoso J, Oliva Domínguez M, Martínez-Beltrán J, Pedraza Cezón L, Moreno Guillén S. Evaluación de un programa de asesoría en terapia antibiótica. Rev Clin Esp 2002; 202: 78-83.

3. López-Medrano F, San Juan R, Serrano O, Chaves F, Lumbreras C, Lizasoain M, et al PACTA: Efecto de un programa no impositivo de control y asesoramiento del tratamiento antibiótico sobre la disminución de los costes y el descenso de ciertas infecciones nosocomiales. Enferm Infecc Microbiol Clin 2005; 23: 186-90.

4. Marr JJ, Moffet HL, Kunin CM. Guidelines for improving the use of antimicrobial agents in hospitals: a statement by the Infectious Diseases Society of America. J Infect Dis 1988; 157: 869-76.

5. Gould IM. Antibiotic policies and control of resistance. Curr Opin Infect Dis 2002; 15: 395-400

6. Gould IM. A review of the role of antibiotic policies in the control of antibiotic resistance. J Antimicrob Chemother 1999; 43: 459-65.

7. World Health Organization: Guidelines for ATC classification and DDD assignment. Oslo: Norsk Medisinaldepot; 1996.

8. Shlaes DM, Gerding DM, John JF, Craig WA, Bornstein DL, Duncan RA, et al. Society for Healthcare Epidemiology of America and Infectious Diseases Society of America Joint Committee on the Prevention of Antimicrobial Resistance: guidelines for the prevention of antimicrobial resistance in hospitals. Clin Infect Dis 1997; 25: 584-99.

9. McGowan JE. Do intensive hospital antibiotic control programs prevent the spread of antibiotic resistance? Infect Control Hosp Epidemiol 1994; $15: 478-83$

10. White AC, Atmar RL, Wilson J, Cate TR, Stager CE, Greenberg SB. Effects of requiring prior authorization for selected antimicrobials: Expenditures,

\section{AGRADECIMIENTOS}

A los servicios de Admisión y Documentación Clínica (Dr. Arturo Ramos Martín-Vegué), Cirugía General, Cirugía Ortopédica y Traumatología, Neurocirugía y Cirugía Cardiovascular del Hospital Universitario Puerta de Hierro por su colaboración en este trabajo. susceptibilities, and clinical outcomes. Clin Infect Dis 1997; 25: 230-9.

11. Landman D, Chokalingam M, Quale JM. Reduction in the incidence of methicillin-resistant Staphylococcus aureus and ceftazidime-resistant Klebsiella pneumoniae following changes in a hospital antibiotic formulary. Clin Infect Dis 1999; 28: 1062-6.

12. Fraser GL, Stogsdill P, Dickens JD, Wennberg DE, Smith RP, Prato BS Antibiotic optimization. An evaluation of patient safety and economic outcomes. Arch Intern Med 1997; 157: 1689-94.

14. Byl B, Clevenbergh P, Jacobs F, Struelens MJ, Zech F, Kentos A, et al. Impact of infectious diseases specialists and microbiological data on the appropriateness of antimicrobial therapy for bacteremia. Clin Infect Dis 1999; 29: 60-6.

15. MacGregor RR, Graziani AL. Oral administration of antibiotics: a rational alternative to parenteral route. Clin Infect Dis 1997; 24: 457-67.

16. Fraser GL, Stogsdill P, Dickens JDJ, Smith RP. Antibiotic optimization: An evaluation of safety and economic outcomes. Arch Intern Med 1997; 157: 1689-94.

17. Bantar C, Sartori B, Vesco E, Heft C, Saúl M, Salamone F, et al. A hospital wide intervention program to optimize the quality of antibiotic use: Impact on prescribing practice, antibiotic consumption, cost savings, and bacterial resistance. Clin Infect Dis 2003; 37: 180-6.

18. Abad F, Calbo F, Zapater P, Rodríguez-Vilanova F, García-Pérez L, Sacristán JA. Comparative pharmacoeconomic study of vancomycin and teicoplanin and in intensive care patients. Int J Antimicrob Agents 2000; 15: 65-71.

19. Laing RBS, Mackenzie AR, Shaw H, Gould IM, Douglas JG. The effectof intavenous-to-oral switch guidelines on the use of parenteral antimicrobialsin medical wards. J Antimicrob Chemother 1998; 42: 107-11.

20. Vogel F. Sequential therapy in the hospital management of lower respiratoryinfections. Am J Med 1995; 99: S14-S9. 